\title{
El control de gestión en el antiguo MOPT. Los límites de una visión gerencial
}

José M. Giró Roca*

\section{Introducción}

La aceptación generalizada conseguida por las funciones de Control de Gestión en las empresas, públicas y privadas, contrasta con la escasez de funciones análogas existentes en el seno de las Administraciones Públicas, a pesar del protagonismo que han asumido en la oferta de bienes y servicios a los ciudadanos-consumidores. Las razones que pueden explicar esta diferencia son múltiples y complejas. Después de una breve referencia histórica al concepto de control, se resumen algunas de las reflexiones que condicionaron un intento de transferir al sector público métodos diseñados para garantizar una gestión eficaz en las grandes empresas.

Con el desarrollo de la teoría de sistemas durante la segunda mitad de este siglo, el concepto de control ha evolucionado desde una concepción muy próxima a la simple verificación del correcto cumplimiento de las normas establecidas, hasta el estudio de las desviaciones de los resultados obtenidos en comparación con los objetivos previamente establecidos; del análisis de sus causas y de la determinación de las acciones correctoras que deberían adoptarse para obtener los resultados deseados. En definitiva, el concepto cibernético del ciclo de control.

A pesar de que el desarrollo formal de la citada teoría de sistemas está históricamente relacionado con el control de los procesos industriales primero y de los sofisticados sistemas de navegación aérea y espacial, después, su ámbito de aplicación se ha extendido rápidamente al llamado mundo del management, otra de las grandes innovaciones de este siglo. De esa forma, los antiguos dueños se han convertido en gestores (managers) y la dirección de los negocios se ha llegado a confiar a personas más formadas para ejercer una función directiva que conocedoras de los pormenores que caracterizan una determinada actividad empresarial.

Cuando P. Druker escribió The Practice of Management en los años cincuenta, en plena controversia sobre el impacto de la automatización en la economía productiva, invitaba a considerar a la empresa como un todo interdependiente, es decir, un sistema, e introducía el concepto cibernético de control como una parte integrante de la función directiva, pero sin anticipar, al menos de forma explícita, la necesidad de crear un área funcional especializada en el cierre del ciclo de realimentación, es decir, en el Control de Gestión.

Veinte años después, en 1973, el mismo autor dedica al Control un capítulo entero de su obra Management, Tasks, Responsibilities and Practices. Mientras controles, en plural, los considera sinónimos de mediciones e información, el control, en singular, lo trata como una componente más de las funciones directivas. Los controles se relacionan con los medios, dice, el control con el fin. Y añade : El control se ocupa de las expectativas, es decir, el futuro. Los controles son analíticos y se interesan en lo que era y en lo que es.

A partir de la década de los 70 , el concepto de control, obviamente en su acepción cibernética, aparece en la literatura especializada como una parte indisociable de la función directiva. La forma en que se ejerza en las organizaciones y su contenido preciso dependerán de muchos factores, pero nadie pone en duda que la dirección de una actividad empresarial compleja no puede realizarse eficazmente sin los necesarios instrumentos de Control de Gestión.

Durante el último cuarto de siglo hemos asistido a la generalización de los conceptos de planificación estratégica y a la espectacular proliferación de sistemas de información capaces de dar un soporte eficaz a los procesos de toma de decisiones. De esta forma, la función directiva se ha consolidado como una labor en equipo, capaz de compartir, no sólo la coordinación de los planes de acción establecidos, sino la elección misma de los objetivos y de las estrategias más adecuadas para conseguirlos. El Control de Gestión, por su parte, se ha consolidado como una función garante de la coherencia de entre planes estratégicos y el control de las operaciones diarias.

En el ámbito de las administraciones se observa la existencia de movimientos culturales similares alrededor de los conceptos básicos de la Dirección (Planificación, Liderazgo, Organización y Control entre los más destacados), pero con un grado de introducción mucho menos generalizado, probablemente debido a la dificultad de adecuar al ámbito de lo público algunos de los principios en los que se descansa la gestión de las empresas privadas. 
A lo largo de la páginas que siguen, se analizan las dificultades y dudas surgidas al tratar de desarrollar un modelo de Control de Gestión en el ámbito del entonces Ministerio de Obras Públicas y Transportes.

\section{Una Dirección General de Control de Gestión} en la Administración Pública Española

Con la creación del Ministerio de Obras Públicas y Transportes por la fusión del antiguo Deparamento de Obras Públicas y Urbanismo con el de Transportes, Turismo y Comunicaciones, se presenta la oportunidad de mejorar la coordinación entre la construcción de los diferentes tipos de infraestructuras y su explotación, desarrollando sistemas de seguimiento y control que permitan incorporar a las reflexiones estratégicas toda la información recopilada al controlar las operaciones $y$, al mismo tiempo, extender, a la fase de control, los modelos y sistemas utilizados para diseñar el futuro.

El Real Decreto 1125/1991, de 22 de julio, de reestructuración de la Subsecretaría de Obras Públicas y Transportes, desarrolla la estructura orgánica de la Dirección General de Sistemas de Información y Control de Gestión y Procedimientos de acuerdo con las líneas directrices y criterios establecidos por el Real Decreto 576/1991, de 21 de abril, por el que se estableció la estructura orgánica básica del Ministerio de Obras Públicas y Transportes. Las funciones de esta Dirección General, de nueva creación, corresponden a las asignadas a la Subsecretaría en el ámbito del Control de la actividad del Departamento, en los aspectos financieros, de gestión y de procedimientos, con el fin de poder evaluar el funcionamiento, la eficacia y el rendimiento del personal y servicios del Ministerio, sin perjuicio de las actividades que en estas materias pueda efectuar la IGAE, de acuerdo con la normativa entonces vigente.

Podría interpretarse que al reunir la funciones informáticas, de control de eficacia, de inspección y de procedimientos en una nueva Dirección General se reconoce que la complejidad de la construcción de infraestructuras y su posterior explotación exige la creación de un órgano responsable de la función de control, de la misma forma que ya no se concibe la gestión eficaz de un sistema complejo sin una estructura formal de feed-back.

Desde el punto de vista de la organización de la Administración Pública, este organismo de control obedecería a la llamada causa organizativa del control, es decir, a la necesidad de resolver las dificultades surgidas como consecuencia de la distancia existente entre quien tiene la autoridad para decidir y los que deben ejecutar o cumplir estas decisiones. Este control resulta tanto más necesario cuanto más grandes y complejas sean las organizaciones y más numerosas y diferentes las unidades que desarrollan su actividad. (Ver la Ponencia presentada por el Interventor General del Estado en las jornadas para la Modernización de las Administraciones Públicas, de Julio de 1990). Independientemente de la solidez de las muchas razones que justifican poner en duda la validez de una transferencia sistemática de técnicas y métodos entre los llamados mundo público y mundo privado, parece evidente que es aconsejable construir modelos organizativos basados en la amplia experiencia desarrollada por las grandes empresas para dar adecuada respuesta a la citada causa organizativa del control, que se manifiesta en el mundo privado de forma muy similar a la observada en las Administraciones Públicas.

Si el Control de Gestión debe entenderse como el proceso mediante el cual la dirección trata de asegurarse que los recursos se obtienen y se usan eficazmente para el logro de los objetivos organizacionales (ANTHONY, 1968) al agrupar en una misma unidad las funciones de inspección, los sistemas de información, los procedimientos y el control de las operaciones se ha dotado al departamento de una unidad responsable de completar el ciclo de feed-back citado anteriormente, puesto que sus objetivos corresponden a las funciones asignadas al citado ciclo en los sistemas complejos.

Debe recordarse, en este punto, que la validez del modelo cibernético aplicado a las organizaciones humanas presenta importantes limitaciones (RICART), entre las que cabe destacar la inexistencia de situaciones repetitivas que permitan validar los modelos utilizados y la imposibilidad de establecer mecanismos del tipo causa-efecto que sean aplicables a los comportamientos de las personas que constituyen los equipos humanos de las organizaciones. Hecha esta salvedad, el control de gestión deberá establecer los procesos de realimentación indicados en la Figura 1, una vez adaptados a las particularidades de las Administraciones Públicas y las propias del Departamento.

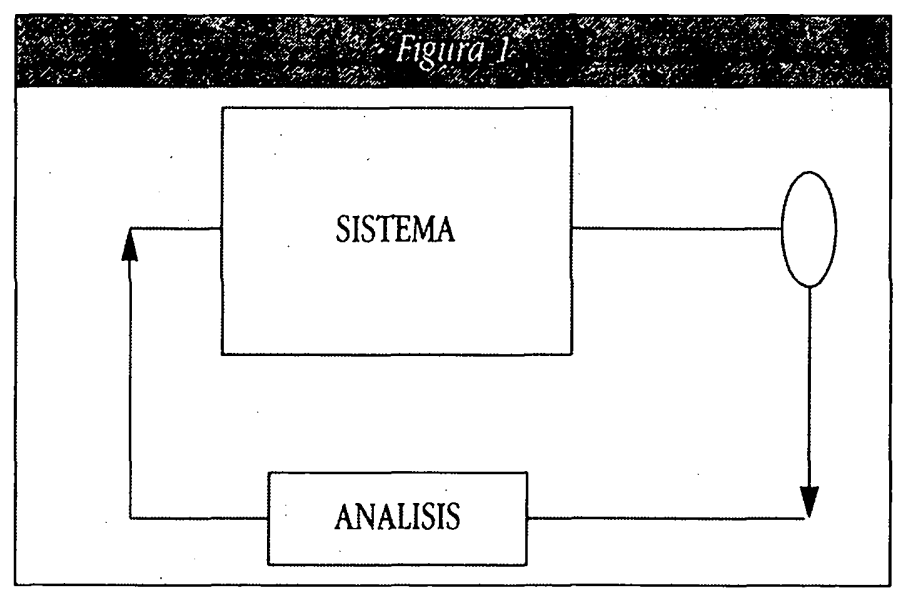




\section{Control de gestión} y planificación estratégica

La creación de una Secretaría General de Planificación en el seno del Ministerio de Obras Públicas y Transportes, en 1991, puede considerarse una consecuencia inmediata del concepto organizativo subyacente en la concepción del Departamento, comparable a la visión organizativa de las grandes empresas que han optado por la creación de departamentos especializados en la planificación estratégica. En las organizaciones empresariales, sin embargo, la función de planificación (componente tan intrínseca de la función directiva como el control) ha sido aislada como una actividad independiente, muchos años después de haberse desarrollado plenamente la función de control, en el sentido expresado anteriomente.

De hecho, las unidades especializadas en planificación se han generalizado a medida que se ha sistematizado el análisis de las estrategias competitivas en la segunda mitad de este siglo y, en muchos casos, se han desarrollado a partir de las funciones de Control de Gestión con lo que Planificación y Control han podido incluso ser integrados en una misma unidad organizativa, responsable del desarrollo metodológico de ambas funciones.

En las Administraciones públicas, este proceso, que podría calificarse como natural, resulta difícilmente reproducible porque sus sistemas presupuestarios y contables no han sido concebidos para ejercer un tipo de control que precisa combinar los datos económicos con los indicadores físicos de actividad, de forma que se puedan analizar las desviaciones respecto a unos objetivos necesariamente definidos como una combinación de magnitudes de muy diversa naturaleza.

A modo de ejemplo, los planes de inversión de un Departamento tienen una creciente lógica global, a largo plazo, debido a las interdependencias entre los distintos sectores económicos y sus correspondientes entornos. Un plan de carreteras, pongamos por caso, se diseña con una visión a largo plazo y en el momento de su aprobación tiene la coherencia que corresponde a los objetivos con los que ha sido desarrollado, expresados en unidades físicas de realización, mejora en los servicios ofrecidos a los ciudadanos medidos en términos de conectividad, por ejemplo, y basados en un cierto equilibrio coste-beneficio, no reflejados en los Presupuestos del Estado.

Una vez el plan se traduce a actuaciones y sus correspondientes fases sufren las vicisitudes propias de su largo período de ejecución, aparecerán desviaciones respecto a los hipótesis iniciales que pueden producir la pérdida de la coherencia inicial para algunas de las actuaciones realizadas.

La Figura 2 ilustra como un plan inicialmente bien concebido, pudo finalmente ser percibido por parte de los ciudadanos-usua- rios como una actuación censurable, como consecuencia de las decisiones que fue preciso tomar en la fase de ejecución. La excelente idea de enlazar la nueva autovía T-C con la L-B, por medio del tramo $C-B$, con el fin aprovechar la inminente construcción del tramo B-A para enlazar eficazmente $T$ y $M$ sin necesidad de afrontar el elevado coste de la construcción del tramo C-G-M, se convierte en una actuación incomprensible si $\mathrm{C}$-B se abre al público dos años antes de haber completado la remodelación de A-B. En estas condiciones, los vehículos que partiendo de T son invitados a dirigirse a $\mathrm{M}$ por el tramo $\mathrm{C}-\mathrm{B}$, se verán atrapados en $\mathrm{B}-\mathrm{A}$ y lamentarán no haber continuado su marcha por la vía C-G-M que, aun siendo convencional, no está bloqueada por el flujo que transita por L-B en dirección al mismo destino final, $M$.

Cuando se construyen simultáneamente varias decenas de tramos con sus correspondientes restricciones de diseño, es inevitable que se produzcan incidencias que alteren los planes iniciales. En la medida que los instrumentos de control sean capaces de establecer relaciones eficaces entre los gastos y el valor generado, situaciones como la descrita serán inmediatamente identificadas, si se producen, y paliadas en los términos que sea posible. En el mundo empresarial, las inversiones y los gastos son siempre confrontados a los ingresos que producen y las desviaciones respecto a los presupuestos iniciales analizadas a partir de los indicadores físicos de actividad. De esta forma, la pérdida producida por un activo con baja rotación (como sería el caso del tramo B-C en el supuesto contemplado) es rápidamente identificada. Los modernos sistemas de gestión se basan en actualizaciones permanentes de los resultados previsibles a medio plazo, lo que exige un continuo control de los desvios observados en los planes de actuación, en sus aspectos fisico y económico, lógicamente integrados en un único sistema de información y control De esta forma, es posible proponer las acciones correctoras necesarias para alcanzar los objetivos establecidos o reformularlos, llegado el caso, (ciclo cibernético de control).

Los diversos niveles de los ciclos de control facilitan el establecimiento de relaciones más estrechas entre Estrategia, Planes, Programas, Presupuestos y Ejecución, integrándolos en un complejo sistema de gestión basado en sistemas de información para la toma de decisiones. De esta forma, las empresas consiguen ser más competitivas en un mundo progresivamente globa-

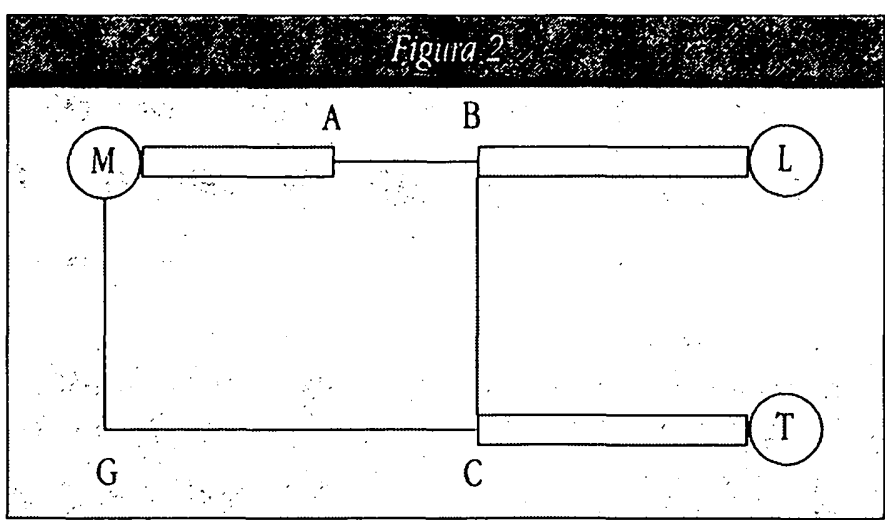


lizado y, sin embargo, fuertemente orientado a los resultados a corto plazo, lo que exige un eficaz control de las operaciones diarias para poder aprovechar las oportunidades ofrecidas por los mercados y, al mismo tiempo, ejecutar eficazmente los planes establecidos a partir de las estrategias a largo plazo.

Como consecuencia de la integración, en el antiguo MOPT, de las funciones informáticas en una nueva unidad de Control de Gestión, el desarrollo de un sistema informatizado de gestión de expedientes de inversión, inicialmente concebido como un instrumento destinado a ordenar los documentos administrativos que acompañan a las actuaciones inversoras, se extendió al ámbito del control y la planificación, dotándolo de las funcionalidades necesarias para calcular las necesidades de recursos a medio plazo y proyectar las consecuencias previsibles de las desviaciones observadas.

De esta forma, situaciones como la descrita en la Figura 2 podrían ser tratadas adecuadamente. Es evidente que seguirán presentándose incidencias imprevisibles capaces de desvirtuar la lógica de un plan. Sin embargo, mediante un adecuado sistema de control se dispondrá de la observabilidad necesaria para optimizar la gestión del sistema a corto y medio plazo. En el caso que nos ocupa esto significaría desde vincular la ejecución del tramo C-B al progreso del B-A (Control Operativo), hasta la modificación de la normativa que impide retrasar la ejecución de obras ya iniciadas para poder transferir parte de su presupuesto a otras de mayor interés a corto plazo, como podría hacer cualquier empresa privada si le conviniera económicamente.(Control Estratégico). El éxito empresarial no es independiente de la capacidad de gestionar adecuadamente las prioridades en cada momento, baciendo compatibles las decisiones estratégicas con las oportunidades del momento.

En un contexto de recursos económicos tan limitados como los sometidos a la disciplina de los Presupuestos del Estado, un sistema de control capaz de poner en evidencia el elevado coste de una utilización sub-óptima de los recursos disponibles permitiría que los procesos reguladores se realizaran con un conocimiento preciso de las consecuencias económicas de las exigencias de la normativa establecida. Se trata, en definitiva, de reproducir en el mundo puiblico el debate también frecuente en las grandes empresas, entre procedimientos, controles y resultados. En la actualidad, la escasez de medios capaces de establecer relaciones sólidas entre las desviaciones observadas, sus causas y las alternativas desechadas, impide que los ciudadanos (accionistas y clientes de lo público, al mismo tiempo) dispongan de los medios necesarios para que sus representantes electos puedan establecer un compromiso económicamente razonable entre los valores de una normativa exigente y los costes de oportunidad asociados a las restricciones que establezca.

\section{El diseño de un sistema de control. Organización, objetivos y resultados}

Todo sistema de control de gestión en una organización precisa que se definan tres elementos básicos:

- Los objetivos de la organización, alrededor de los cuales se deberá diseñar su estructura y ordenar su actividad.

- Los sistemas de medida de los resultados y del control de las desviaciones que permitirán realizar un diagnóstico de su situación.

- Las acciones correctoras o modelos de decisión que deberán aplicarse para reconducción de la evolución del sistema hacia sus objetivos.

Los organismos públicos constituyen una categoría de entidades en las que la definición de objetivos y resultados resulta particularmente difícil, incluso si se consigue evitar el permanente debate sobre validez de estos conceptos en el ámbito de lo público recurriendo a posturas tan pragmáticas como la sugerida por J.L. DeLIGNY al reproducir la recomendación de unos funcionarios japoneses: no se trata de que discutan si es o no posible trasladar los conceptos de gestión del mundo privado al público, para conseguir una administración más eficaz y eficiente, se trata, simplemente, de que el primer país que lo consiga ganará la batalla de la competitividad, en un mundo cada vez más globalizado.

Cediendo pues al pragmatismo y aceptando la validez de unos conceptos similares a los de objetivos y resultados para determinadas funciones públicas, se podrán diseñar sistemas de control capaces de transmitir a los ciudadanos una imagen fiel del buen funcionamiento de sus instituciones públicas a través de su percepción del valor obtenido de los servicios recibidos. El concepto de valor podría definirse, como propone M. PORTER, como lo que los clientes estén dispuestos a pagar, es decir, recordando la importancia de evitar las frecuentes confusiones entre coste, esfuerzo realizado y valor realmente percibido por los usuarios.

Las grandes empresas suelen optar por segmentar sus actividades en unidades de negocio cuando tienen dificultades para establecer sistemas de objetivos capaces de relacionar eficazmente la calidad de la actuación de sus gestores con las apreciaciones de los consumidores en los distintos segmentos de mercados y con los intereses de los accionistas.

De forma análoga, será preciso segmentar el análisis de resultados de la función pública para conseguir establecer sistemas de control que permitan optimizar la creación de valor percibido por los clientes, siempre que la complejidad del ámbito de actuación o 
la mulicidad de objetivos impida definir un sistema único de evaluación. Tomando a RENFE como ejemplo, a pesar de que no sea un organismo muy representativo de las funciones públicas porque su actividad es de tipo empresarial, puede utilizarse como prueba de que al segmentar una actividad en unidades de negocio se pudieron establecer objetivos basados en resultados medibles y, como consecuencia, tomar decisiones de optimización de recursos dificilmente identificables utilizando un modelo globalizado.

En la Figura 3 se reproduce un esquema similar al realmente utilizado para evaluar la viabilidad estratégica de un determinado servicio y ubicar, en su justo lugar, el debate de la eficiencia. El gráfico indica qué precio sería necesario establecer por viajero y kilómetro recorrido en un determinado tipo de tren nocturno, que recorriera diariamente los kilómetros indicados, para conseguir equilibrar los ingresos y los gastos de explotación, en los que no se incluyen los de las infraestructuras utilizadas para simplificar el análisis.

Evidentemente, el punto de equilibrio dependerá de la eficiencia interna de la organización ferroviaria. Olvidando por un instante este hecho, si los datos utilizados fueran correctos, debería concluirse que son necesarios recorridos diarios superiores a $800 \mathrm{Km}$, con una ocupación media del $70 \%$ para poder ofrecer precios no superiores a $5 \mathrm{pts} / \mathrm{Km}$ sin sufrir pérdidas de explotación. Teniendo en cuenta que la distancia de Madrid a cualquiera de las capitales de provincia es inferior a este mínimo, con la tecnología utilizada en este caso no sería posible equilibrar la cuenta de resultados de este servicio nocturno si se pretendiera cobrar un precio competitivo con los ofrecidos por otros medios alternativos que ofrecen tarifas como la indicada. Visto que los precios de equilibrio necesarios para los trayectos de $600 \mathrm{Km}$ se aproximan a las 9 pts por Viajero-Km para ocupaciones cercanas al 50\%, no es razonable esperar que un aumento de la eficiencia permita compensar por completo los problemas estructurales y alcanzac la rentabilización de la explotación.

A pesar de que los datos ofrecidos no corresponden a la situación actual de este tipo de explotación, el modelo propuesto no es conceptualmente muy diferente del tipo de análisis que se realizan habitualmente con los métodos propios del Control de Gestión, ni las conclusiones muy distintas de las observadas en determinados servicios públicos. Debido al interés social de las actuaciones públicas y a la inexistencia de mercados en los que poder definir claramente los conceptos de resultado, producto, y clientes resultará imposible, en muchos casos, definir sistemas de control de gestión que permitan dar una respuesta convincente a los niveles de eficacia exigidos por los ciudadanos que juzgan el resultado según su particular percepción del valor recibido como clientes $y / 0$ accionistas. Sin embargo, mediante una utilización generalizada del concepto de control de gestión en los distintos niveles de responsabilidad existente en las instituciones públicas, es posible llegar a construir una serie de indicadores que permitan responder a las inquietudes planteadas de forma parcial, pero convincente. Mediante su utilización sistemá-

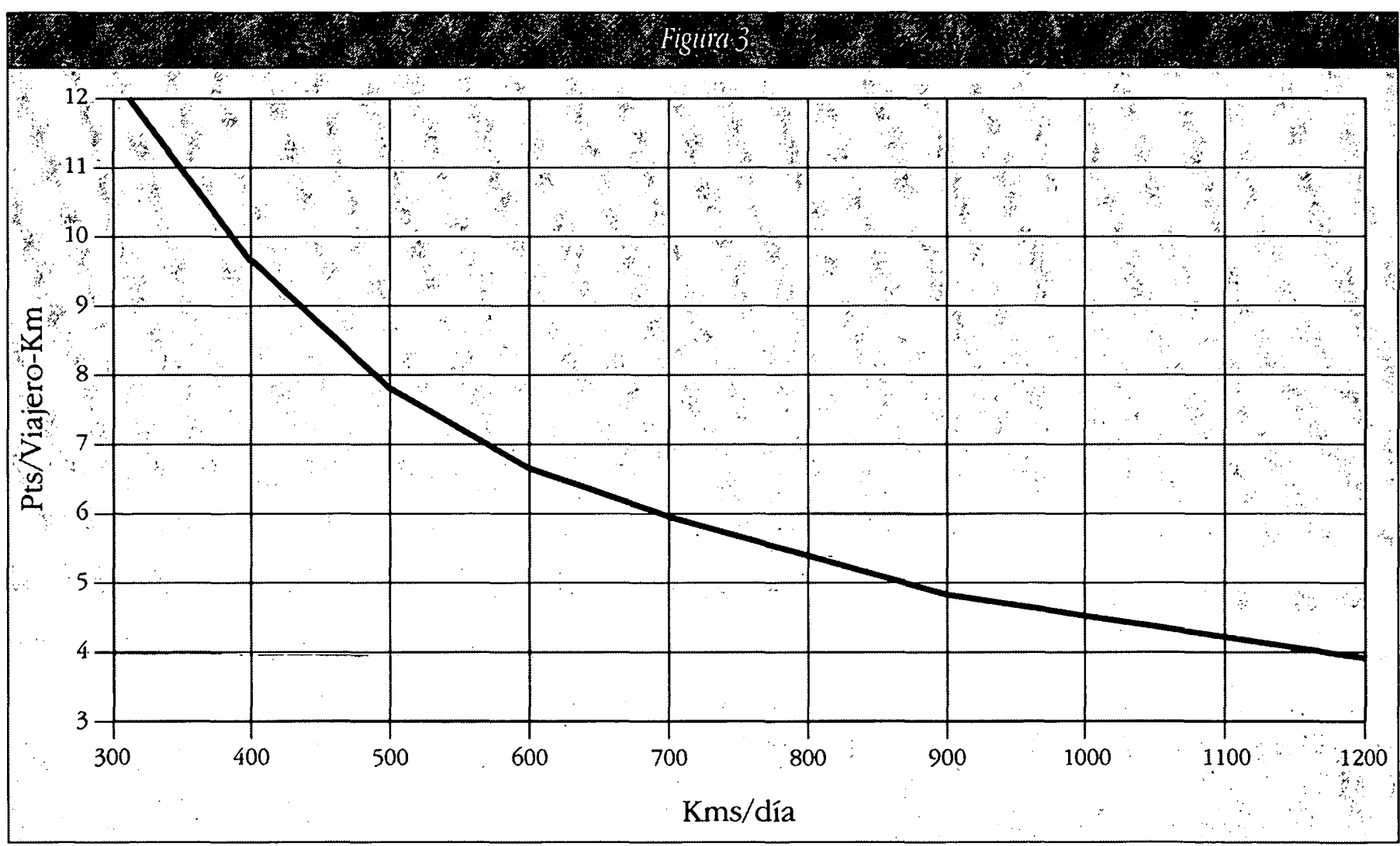


tica se llegarán a diseñar, por aproximaciones sucesivas, sistemas de control de ámbito global.

Como se ha dicho al principio, los modelos de planificación estratégica actualmente utilizados en el mundo empresarial, se han desarrollado después de muchos años de controlar los resultados y analizar las actividades por segmentos buscando las claves del éxito y del fracaso. Es muy difícil encontrar historias de éxito que no tengan su inicio en un cierto proceso inductivo. Cuanto más se retrase la introducción de sistemas eficaces de medición de algún tipo de resultado en el ámbito de las Administraciones Públicas -siempre que sean comprensibles por los accionistas-usuarios aunque sean conceptualmente cuestionables- más difícilmente se obtendrá su apoyo efectivo en el desarrollo de planes estratégicos.

Los sistemas de Control de Gestión en las empresas han contribuido de una forma notable a la calidad de la gestión presupuestaria y a la fiabilidad de las previsiones de resultados. De esta forma los accionistas y sus consejos de administración pueden otorgar a los gestores un amplio grado de autonomia y libertad de acción en el corto y medio plazo, respetando los acuerdos estratégicos plasmados en un presupuesto puntualmente seguido a lo largo del ejercicio económico. De esta forma, los accionistas no suelen dar muestras de intranquilidad permanente y desconfianza bacia los resultados publicados por las empresas, lo que permite que sus directivos actüen con la estabilidad necesaria para asumir sus responsabilidades, incluso en condiciones de extrema competitividad.

La extensión de cuanto antecede al ámbito de actividad de una Administración pública más próxima a las funciones reguladoras presenta, sin embargo, dificultades particulares que Jerome Dupuls resume en los tres apartados siguientes:

\section{- Diversidad de Objetivos.}

La existencia de una gran cantidad de objetivos muy diversos que caracteriza a cualquier actividad pública hace extremadamente difícil establecer una estructura organizativa basada en la delegación. Como, además, una parte importante de estos objetivos tienen un origen externo, los resultados de sus actuaciones sólo pueden ser analizados desde el punto de vista del conjunto del sistema público. Cada organismo realiza actividades cuyos efectos sobrepasan ampliamente el marco de actuación que justifica su propia existencia.

- Centros de responsabilidad.

La lógica tradicional de las organizaciones públicas descansa fundamentalmente en una obligación de ofrecer unos medios mientras que la gestión por centros de responsabilidad parte de la existencia de una obligación de resultados, cuya definición exige partir de una estructura de objetivos.

\section{- Mercado y productos.}

La ausencia del concepto de mercado dificulta la elección de indicadores que permitan elegir los productos a ofrecer con la precisión que pueden hacerlo las empresa privadas. En consecuencia, una buena parte de la labor propia de los responsables del control de gestión de los organismos públicos consiste en identificar cuáles son los productos sobre los que podrán construirse los sistemas de gestión. Por ello, herramientas como la contabilidad analítica, plenamente identificadas con la lógica de su medio de origen, que es el mundo privado, no pueden ser fácilmente transferidos a las Administraciones públicas. En realidad, se trata de transferir la lógica de un método de gestión nacido en el ámbito de lo privado y que es deseable incorporar al mundo público, por medio de un esfuerzo pragmático de adaptación.

Es precisamente esta voluntad de pragmatismo la que permitió que en el ámbito del antiguo MOPT se desarrollara un análisis del contenido de todas las funciones de algunos centros directivos asignando porcentajes de tiempo dedicado a cada actividad y calculando las horas-funcionario necesarias para realizar la mayor parte de las actividades que les correspondían. Una vez ubicados los tiempos obtenidos en sendas tablas ordenadas por unidades funcionales $y$ tareas relizadas se pudieron analizar las redundancias, las imprecisiones del sistema de asignación de responsabilidades y definir, incluso, algunos indicadores de productividad que, a falta de mejor patrón de referencia, se compararon con ellos mismos en otros períodos de tiempo.

Como es obvio, a partir de este tipo de análisis pueden establecerse indicadores de actividad basados en magnitudes físicas que permitan cuantificar las plantillas necesarias en función de la carga de trabajo previsible, una vez consensuado un cierto reparto de los recursos entre fijos y variables. Partiendo de una fuerte convicción, como la expresada por J. Dupuis, es posible explotar este tipo de modelo y de su propio uso continuado establecer un sistema de Control de Gestión capaz de proteger los intereses del colectivo, en la medida que facilite objetivar la siempre difícil discusión de los medios necesarios para una determinada carga de trabajo.

\section{Conclusión}

Como consecuencia de la difusión generalizada de las tecnologías de la información y la comunicación, nuestra sociedad es cada vez más observable y los ciudadanos tenderán a medir, cada vez con más precisión, la relación existente entre el valor percibido por los servicios recibidos y su coste, independientemente de la titularidad del organismo que lo ofrezca. 
Los mercados han exigido a las empresas que sus sistemas de producción fueran cada vez más competitivos. Actualmente, es dificil imaginar una empresa capaz de permanecer en el mercado sin disponer de los medios necesarios para planificar su actividad, normalmente diseñados a partir de los conocimientos adquiridos gracias al uso continuado de sistemas de control de gestión.

En el ámbito de las Administraciones públicas, sin embargo, los complejos expedientes que circulan de unas secciones a otras para cumplir los trámites necesarios, aparecen en las mesas de los despachos sin un plan previo conocido y sin tener en cuenta la carga de trabajo pendiente en cada unidad administrativa. Además, los criterios de urgencia permanecen ocultos, incluso después de estudiar su contenido, porque los conflictos de prioridades en las ejecuciones de los proyectos no pueden transmitirse manualmente a las tareas administrativas asociadas. De hecho, salvo los casos realmente urgentes, las prioridades de resolución de los trámites administrativos pueden ser definidas por el caprichoso azar introducido por los sistemas de correo interno.

Al diseñar el sistema informático de gestión de expedientes citado anteriormente, se pudo adecuar el orden de ejecución de trámites sugerido por el ordenador a los criterios de prioridad establecidos por el plan global de proyectos en curso. Es como si los expedientes urgentes, sepultados en una pila de documentos, fueran capaces de protestar airadamente, ante los atónitos ojos de funcionario, por el perjuicio que se está produciendo al no atenderlos de inmediato. De esta forma, la organización de las tareas podría adquirir las características de una fábrica de expedientes gestionada por un GPAO (Gestión de Producción Asistida por Ordenador) capaz de calcular, además, las necesidades de recursos en determinadas fases. Por ejemplo, si las siempre inevitables restricciones de fin de ejercicio tienden a concentrar un exceso de expedientes en las dependencias de la Intervención Delegada, las consecuencias previsibles pueden ser conocidas con antelación suficiente para poder tomas las medidas adecuadas.
Con la tecnología actualmente disponible y la capacidad de las redes de comunicación existentes, las Administraciones públicas podrian ofrecer a sus clientes, por ejemplo, sistemas de intercambio electrónico de datos para la realización de determinados trámites a costes inferiores al del desplazamiento a las oficinas del correspondiente servicio. Estos sistemas podrían permitir, además, consultar el estado de un determinado expediente de forma que pudiera ejercerse un eficaz seguimiento de su progreso en el seno del organismo responsable de su tramitación.

En los años sesenta, las empresas disponían de la tecnología necesaria para aplicar sofisticados sistemas de apoyo a la toma de decisiones que a menudo fueron abandonados por la escasez de datos y el coste de la capacidad de cálculo. Desde el mismo momento en que desaparecieron estas restricciones se produjo una espectacular difusión de los métodos anteriormente diseñados. Actualmente, la más insignificante explotación ganadera, por ejemplo, utiliza sofisticados modelos matemáticos para ser competitiva, optimizando el uso de sus recursos.

Las Administraciones Públicas disponen de los medios necesarios, los modelos están desarrollados o son transferibles desde el sector empresarial y, además, una buena parte de la información necesaria está sobre algún soporte informático. Es cierto que hay ciertas dificultades conceptuales y que siempre se podrán aducir razones de respeto riguroso a los principios que inspiran a la función pública para posponer sistemas más orientados al resultado. ¿Será, entonces, necesaria una introducción generalizada de sistemas de Control de Gestión para crear las condiciones necesarias para que se utilicen, en la práctica, cuantos modelos de gestión superen con éxito el preceptivo análisis coste - beneficio? O habrá que esperar a que las inexorables leyes de la competencia eliminen las barreras existentes, de forma que la búsqueda de la eficiencia y eficacia sea realmente un objetivo prioritario, tal y como precisan los accionistas-clientes para poder sobrevivir en un mundo cada vez más globalizado y competitivo.

Notas

- Ex Director General de Sistemas de Información y Control de Gestión y Procedimientos. MOPT.

\section{Bibliografia}

Jean Louis DeLugY. L'Administration du Future. Eyroles 1990.

Jerome Dupuls. Le contrôle de gestion dans les organisations publiques. Gestión.Puf 1991.

Rober N. ANTHONY. Planning and control systems: a framework for analysis. Harvard-University Press. 1963.
Joan E. RucakT; Jordi Gual; Incentivos y control de la empresa pública. Ariel Economía. 1991.

Peter DRuKER. The practice of Management

Peter DruKer. Management, Tasks, Responsibilities and Practices. 


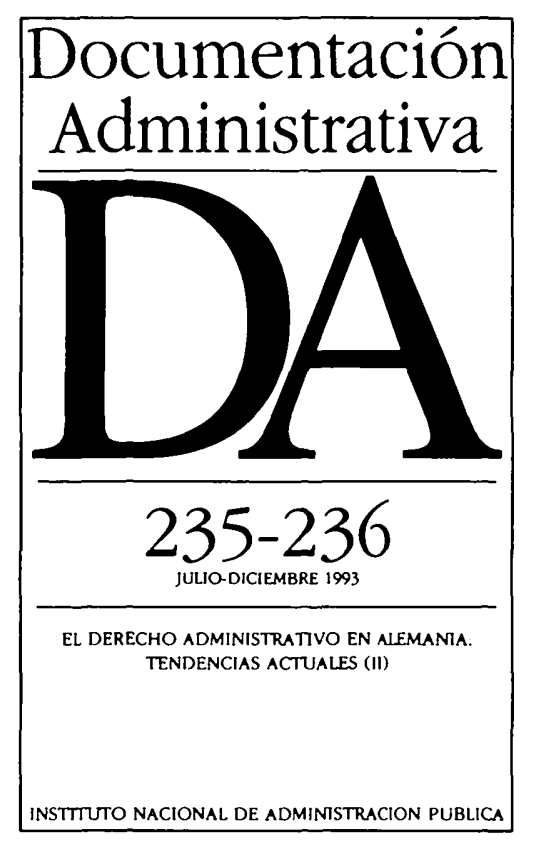

Publicación trimestral del Instituto Nacional de Administración Pública, especializada en Derecho Administrativo y Organizáción y Gestión de las Administraciones Públicas.

Aparecida en 1958, la característica más destacada de su actual etapa es la dedicación monográfica de cáda número a una cuestión especifica, que se analiza desde diferentes puntos de vista por acreditadas firmas de España y otros países.

\section{CONSEJO DE REDACCION}

Presidente: Alejandro Nieto García. Vocales: Manuel Aragón Reyes, Enrique Argullol Murgadas, Miguel Beltrán Villalva, Andrés de Blas Guerrero, José Manuel Castells Arteche, Rafael Gómez-Ferrer Morant, Luis Morell Ocaña, José Ramón Parada Vázquez, Juan Alfonso Santamaría Pastor, Gumersindo Trujillo Fernández.

\section{Director}

\section{Luciano Parejo Alfonso}

\section{Secretario}

Jesús Prieto de Pedro

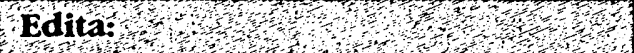

Instituto Nacional de Administración Pública ISSN: 0012-4494

NIPO: 329-94-001-5

Depósito legal: M 581-1958

\section{Distribución y suscripciones:}

Instituto Nacional de Administración Pública Subdirección General de Documentación, Investigación y Publicaciones José Marañón, 12. 28010 MADRID Teléfono: (91) 4461700 Fax: (91) 4450839

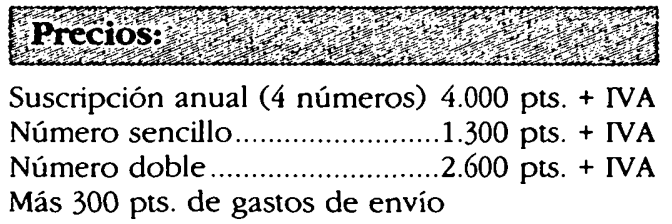

